\title{
THE DEGREE OF REGULARITY OF A QUASICONFORMAL MAPPING
}

\author{
PEKKA KOSKELA
}

(Communicated by Albert Baernstein II)

\begin{abstract}
T. Iwaniec has conjectured that the derivative of a locally $\alpha$-Hölder continuous quasiconformal mapping of $\mathbb{R}^{n}$ is locally integrable to any power $p<\frac{n}{1-\alpha}$. We disprove this conjecture by producing examples of quasiconformal mappings of the plane that are uniformly Hölder continuous with exponent $\frac{1}{2}<\alpha<1$ but whose derivatives are not locally integrable to the power $\frac{1}{1-\alpha}$.
\end{abstract}

\section{INTRODUCTION}

Recall that a homeomorphism $f$ of a domain $D \subset \mathbb{R}^{n}$ onto a domain $D^{\prime} \subset$ $\mathbb{R}^{n}$ is $K$-quasiconformal if $f \in W_{\text {loc }}^{1, n}(D)$ and $\left|f^{\prime}(x)\right|^{n} \leq K J_{f}(x)$ holds a.e. in $D$; here $\left|f^{\prime}(x)\right|$ is the operator norm of the formal derivative $f^{\prime}(x)$ of $f$. We call $f$ quasiconformal if $f$ is $K$-quasiconformal for some $K$. Gehring proved in his celebrated paper [G3] that, in fact, $f \in W_{\text {loc }}^{1, p}(D)$ for some $p=$ $p(n, K)>n$ (in the plane this result is due to Bojarski [B]) whenever $f$ is a quasiconformal mapping of a domain $D \subset \mathbb{R}^{n}$. Gehring and Reich have conjectured [GR; G2; I, 9.1] that a $K$-quasiconformal mapping $f$ belongs to $W_{\mathrm{loc}}^{1, p}(D)$ for all $p<\frac{n K}{K-1} .1$

Since $K$-quasiconformal mappings are locally Hölder continuous [G1] with exponent $\frac{1}{K}$, the familiar Sobolev embedding [GT, Theorem 7.26] indicates that the above local Hölder continuity exponent coincides with the exponent that would be implied by the local integrability of the derivative of $f$ to the power $\frac{n K}{K-1}$. This motivates the following conjecture due to Iwaniec.

Conjecture [I, 9.2]. If a quasiconformal mapping $f$ of a domain $D \subset \mathbb{R}^{n}$ is locally Hölder continuous with exponent $0<\alpha<1$, then $f \in W_{\mathrm{loc}}^{1, p}(D)$ for all $1 \leq p<\frac{n}{1-\alpha}$.

Thus the Sobolev embedding is conjectured to be essentially invertible in the class of quasiconformal mappings regardless of the dilatation $K$ of the mapping in question. Unfortunately, this conjecture, which would yield the Gehring and Reich conjecture, is false.

Received by the editors August 3, 1992 and, in revised form, February 15, 1993.

1991 Mathematics Subject Classification. Primary 30C65.

Key words and phrases. Quasiconformal mapping, Sobolev embedding, global integrability of the derivative.

${ }^{1} \mathrm{~K}$. Astala has recently verified the Gehring and Reich conjecture in the plane. 
Theorem. For each $\frac{1}{2}<\alpha<1$ there is a quasiconformal mapping $f$ of the plane such that $f$ is uniformly Hölder continuous with exponent $\alpha$ but the derivative of $f$ fails to be locally integrable with exponent $\frac{1}{1-\alpha}$.

The above theorem immediately disproves Iwaniec's conjecture for $\frac{1}{2}<\alpha<$ 1 . For the other values, one only needs to observe that our theorem guarantees for any $p>2$ the existence of a plane quasiconformal mapping $f$ that is locally Hölder continuous with exponent $\frac{1}{2}$ and such that the derivative of $f$ fails to be integrable to the power $p$; hence Iwaniec's conjecture fails for $0<\alpha \leq \frac{1}{2}$ as well.

The argument we employ for the proof of our theorem is as follows. First we construct a "bad" quasisymmetric mapping $g$ of the real line onto a von Kochtype snowflake quasicircle (cf. [A; T2, p. 151]). Then, employing results of Tukia [T1], we obtain a quasiconformal mapping $f$ of the plane that extends $g$. This extension will be $C^{1}$-smooth outside $\mathbb{R}$, but the derivative of the extension blows up uniformly when we approach $\mathbb{R}$.

We wish to point out that our construction is fairly standard. Nevertheless, applications of this type seem to have stayed unnoticed; we will employ our construction also in a forthcoming work [KKM] to show sharpness of Radótype theorems for solutions to degenerate elliptic partial differential equations.

\section{Proof of Theorem}

Recall that an embedding $g: \mathbb{R} \rightarrow \mathbb{C}$ is quasisymmetric if there is a constant $C$ such that

$$
|g(x)-g(y)| \leq C|g(x)-g(w)|
$$

whenever $x, y, w \in \mathbb{R}$ satisfy $|x-y| \leq|x-w|$. By the Beurling-Ahlfors extension theorem each quasisymmetric $g$ with $g(\mathbb{R})=\mathbb{R}$ extends to a quasiconformal mapping of the plane. The main ingredient in the proof of our theorem is the following similar extension result due to Tukia [T1].

2.1. Theorem [T1]. If $g: \mathbb{R} \rightarrow \mathbb{C}$ is quasisymmetric, then $g$ has a quasiconformal extension $f: \mathbb{C} \rightarrow \mathbb{C}$ such that $f$ is $C^{1}$ in $\mathbb{C} \backslash \mathbb{R}$ and

$$
C^{-1}\left|f^{\prime}(x+i y)\right| \leq \frac{|f(x+y)-f(x-y)|}{|y|} \leq C\left|f^{\prime}(x+i y)\right|
$$

for some fixed constant $C$ and each $x+i y \in \mathbb{C} \backslash \mathbb{R}$.

Now we construct an appropriate quasisymmetric $g$. This construction appears to be folklore (cf. [A; FM; M; T2, p. 151]). Nevertheless, we sketch the necessary steps for the convenience of the reader. Fix $\frac{1}{4}<t<\frac{1}{2}$, and let $a_{1}=0, a_{2}=t, a_{3}=\frac{1}{2}+i\left(t-\frac{1}{4}\right)^{1 / 2}, a_{4}=1-t, a_{5}=1$. Then the length of each line segment $\overline{a_{j} a_{j+1}}, j=1,2,3$, is $t$. Next, let $\sigma_{j}, j=1, \ldots, 4$, be similarities which map the line segment $\overline{a_{1} a_{5}}$ onto $\overline{a_{j} a_{j+1}}$ with $\sigma_{j}\left(a_{1}\right)=a_{j}$.

For a set $A$ in the plane write $\sum(A)=\bigcup_{1}^{4} \sigma_{j}(A)$, and for $p>1$ set $\sum^{p}\left(\overline{a_{1} a_{5}}\right)=\sum\left(\sum^{p-1}\left(\overline{a_{1} a_{5}}\right)\right)$. Then, for each $p \geq 1, \sum^{p}\left(\overline{a_{1} a_{5}}\right)$ consists of $4^{p}$ line segments $J_{k}$ of length $t^{p}$. Assume that they are in order on $\sum^{p}\left(\overline{a_{1} a_{5}}\right)$ with $0 \in I_{1}$. Write $I_{k}=\left[(k-1) 4^{-p}, k 4^{-p}\right]$, and pick a homeomorphism $h_{p}$ : $[0,1] \rightarrow \sum^{p}\left(\overline{a_{1} a_{5}}\right)$ such that $h_{p}$ is affine in each $I_{k}$ with $h_{p}\left(I_{k}\right)=J_{k}$. Then the mappings $h_{p}$ converge to a homeomorphism $h:[0,1] \rightarrow h([0,1]):=\gamma_{t}$ with 
$h\left(4^{k} x\right)=\left(\frac{1}{t}\right)^{k} h(x)$ whenever $0 \leq x \leq 4^{k} x \leq 1$. Moreover, $\gamma_{t}$ is the unique compact set $A \subset \mathbb{C}$ with $\sum(A)=A$ (see [H]) and $\gamma_{t}$ is the limit of the iterated arcs $\sum^{p}\left(\overline{a_{1} a_{5}}\right)=\sum\left(\sum^{p-1}\left(\overline{a_{1} a_{5}}\right)\right)$ in the Hausdorff metric. In fact, $\gamma_{1 / 3}$ is the familiar von Koch curve and, for all $\frac{1}{4}<t<\frac{1}{2}, \gamma_{t}$ is a snowflake-type curve.

Notice that $t \gamma_{t}$ is a subarc of $\gamma_{t}$ and likewise $\gamma_{t}$ is a subarc of $\frac{1}{t} \gamma_{t}$. Thus, by defining

$$
\gamma=\bigcup_{j \geq 0}\left(\frac{1}{t}\right)^{j}\left(\gamma_{t} \cup\left(-\gamma_{t}\right)\right),
$$

we obtain an arc $\gamma$ through $\infty$. To complete the construction, we set

$$
g\left(4^{k} x\right)=\left(\frac{1}{t}\right)^{k} h(x)
$$

for $k \geq 1$ and $0 \leq x \leq 1$, and define $g(x), x<0$, by symmetry. Then $g$ is a homeomorphism of $\mathbb{R}$ onto $\gamma$, and it is straightforward to check (see the calculations in [M, pp. 102-103]; cf. also [FM]) that

$$
|x-y|^{\alpha} / C \leq|g(x)-g(y)| \leq C|x-y|^{\alpha}
$$

for some constant $C$ for all $x, y \in \mathbb{R}$, where $\alpha=\log \left(\frac{1}{t}\right) / \log 4$. In conclusion, we obtain

2.3. Lemma. For each $\frac{1}{2}<\alpha<1$ there is an embedding $g: \mathbb{R} \rightarrow \mathbb{C}$ such that

$$
|x-y|^{\alpha} / C \leq|g(x)-g(y)| \leq C|x-y|^{\alpha}
$$

for some fixed constant $C$ for all $x, y \in \mathbb{R}$.

Proof of Theorem. Fix $\frac{1}{2}<\alpha<1$, and let $g$ be a mapping as in Lemma 2.3. Then $g$ is quasisymmetric, and hence Theorem 2.1 provides us with a quasiconformal mapping $f$ of the plane extending $g$. Next, from (2.2) and (2.4) we conclude that

$$
\left|f^{\prime}(x+i y)\right| / C \leq|y|^{\alpha-1} \leq C\left|f^{\prime}(x+i y)\right|
$$

for all $x+i y$ in $\mathbb{C} \backslash \mathbb{R}$, where $C$ is a fixed constant. Hence we deduce that $\left|f^{\prime}\right|^{1 /(1-\alpha)}$ fails to be locally integrable.

We are left to verify the uniform Hölder continuity of $f$. Fix points $z_{1}=$ $x_{1}+i y_{1}$ and $z_{2}=x_{2}+i y_{2}$ in the upper half plane. Integrating the estimate

$$
\left|f^{\prime}(x+i y)\right| \leq C|y|^{\alpha-1}
$$

we arrive at

$$
\left|f\left(z_{j}\right)-f\left(x_{j}\right)\right| \leq C \alpha^{-1} y_{j}^{\alpha}, \quad j=1,2 .
$$

By (2.6) we may assume that $y_{1} \geq y_{2}$ and that $z_{2} \notin B\left(z_{1}, y_{1} / 2\right)$. Then

$$
y_{1} \leq 2\left|z_{1}-z_{2}\right| \text {, }
$$

and the desired estimate

$$
\left|f\left(z_{1}\right)-f\left(z_{2}\right)\right| \leq C\left|z_{1}-z_{2}\right|^{\alpha}
$$

for some constant $C$ follows by the triangle inequality from (2.4), (2.7), and (2.8). Analogously, (2.9) holds for $z_{1}, z_{2}$ in the lower half plane, and thus the 
triangle inequality and the continuity of $f$ verify (2.6) for all $z_{1}, z_{2}$ in the plane. The proof is complete.

2.10. Concluding remarks. (1) Tukia [T2] has used the above construction for $t=\frac{1}{3}$ to produce a quasiconformal group that is not isomorphic to a Möbius group. Recently, Semmes [S] has employed a similar construction in a counterexample related to his work with G. David on strong $A_{\infty}$-weights.

(2) The proof of our theorem reveals that some global integrability results arrived at in $[\mathrm{AK}]$ are sharp. We hope to return to this question in the future.

\section{REFERENCES}

[A] K. Astala, Selfsimilar zippers, Holomorphic Functions and Moduli I (D. Drasin, F. W. Gehring, C. M. Earle, I. Kra, A. Marden, eds.), Springer-Verlag, New York, 1988, pp. 61-74.

[AK] K. Astala and P. Koskela, Quasiconformal mappings and global integrability of the derivative, J. Analyse Math. 57 (1991), 203-220.

[B] B. Bojarski, Generalized solutions of a system of differential equations of first order and elliptic type with discontinuous coefficients, Mat. Sb. 85 (1957), 451-503.

[FM] K. J. Falconer and D. T. Marsh, Classification of quasi-circles by Hausdorff dimension, Nonlinearity 2 (1989), 489-493.

[G1] F. W. Gehring, Rings and quasiconformal mappings in space, Trans. Amer. Math. Soc. 103 (1962), 353-393.

[G2] - Open problems, Proc. Romanian-Finnish Seminar on Teichmuller Spaces and Quasiconformal Mappings, Romania, 1969, p. 306.

[G3] $ـ$, The $L^{P}$-integrability of the partial derivatives of a quasiconformal mapping, Acta Math. 130 (1973), 265-277.

[GR] F. W. Gehring and E. Reich, Area distortion under quasiconformal mappings, Ann. Acad. Sci. Fenn. Ser. A I Math. 388 (1966), 1-14.

[GT] D. Gilbarg and N. S. Trudinger, Elliptic partial differential equations of second order, Springer-Verlag, Berlin and Heidelberg, 1983.

[H] J. E. Hutchinson, Fractals and self-similarity, Indiana Univ. Math. J. 30 (1981), 713-747.

[I] T. Iwaniec, $L^{P}$-theory of quasiregular mappings, Quasiconformal Space Mappings. A Collection of Surveys 1960-90 (M. Vuorinen, ed.), Lecture Notes in Math., vol. 1508, SpringerVerlag, Berlin, 1992, pp. 39-64.

[KKM] T. Kilpeläinen, P. Koskela, and O. Martio, On the fusion problem for second order elliptic partial differential equations, preprint, 1993.

[M] J. McKemie, Quasiconformal groups with small dilatation, Ann. Acad. Sci. Fenn. Ser. A I Math. 12 (1987), 95-118.

[S] S. Semmes, Bilipschitz mappings and strong $A_{\infty}$-weights, Ann. Acad. Sci. Fenn. A I Math. 18 (1993), 211-248.

[T1] P. Tukia, Extension of Lipschitz embeddings of the real line into the plane, Ann. Acad. Sci. Fenn. Ser. A I Math. 6 (1981), 89-94.

[T2] A quasiconformal group not isomorphic to a Möbius group, Ann. Acad. Sci. Fenn. Ser. A I Math. 6 (1981), 149-160.

Department of Mathematics, University of JyväSKylä, P.O. BoX 35, SF-40351 JYVÄSKYLÄ, FINLAND

Current address: Department of Mathematics, University of Michigan, Ann Arbor, Michigan 48109

E-mail address: koskela@math.lsa.umich.edu 